\title{
Residual Risk and Its Risk Factors for Ischemic Stroke with Adherence to Guideline-Based Secondary Stroke Prevention
}

\author{
Yuesong Pan, ${ }^{\text {a,b }}$ Zixiao Li, ${ }^{\text {a,b }}$ Jiejie Li, ${ }^{\text {a,b }}$ Aoming Jin, ${ }^{\text {a,b }}$ Jinxi Lin, ${ }^{\text {a,b }}$ Jing Jing, ${ }^{\text {a,b }}$ Hao Li, ${ }^{\text {a,b }}$ Xia Meng, ${ }^{\text {a,b }}$ \\ Yilong Wang, ${ }^{\mathrm{a}, \mathrm{b}}$ Yongjun Wang ${ }^{\mathrm{a}, \mathrm{b}}$ \\ ${ }^{a}$ Department of Neurology, Beijing Tiantan Hospital, Capital Medical University, Beijing, China \\ ${ }^{b}$ China National Clinical Research Center for Neurological Diseases, Beijing, China
}

Background and Purpose Despite administration of evidence-based therapies, residual risk of stroke recurrence persists. This study aimed to evaluate the residual risk of recurrent stroke in acute ischemic stroke or transient ischemic attack (TIA) with adherence to guideline-based secondary stroke prevention and identify the risk factors of the residual risk.

Methods Patients with acute ischemic stroke or TIA within 7 hours were enrolled from 169 hospitals in Third China National Stroke Registry (CNSR-III) in China. Adherence to guideline-based secondary stroke prevention was defined as persistently receiving all of the five secondary prevention medications (antithrombotic, antidiabetic and antihypertensive agents, statin and anticoagulants) during hospitalization, at discharge, at 3,6, and 12 months if eligible. The primary outcome was a new stroke at 12 months.

Results Among 9,022 included patients (median age 63.0 years and 31.7\% female), 3,146 (34.9\%) were identified as adherence to guideline-based secondary prevention. Of all, 864 (9.6\%) patients had recurrent stroke at 12 months, and the residual risk in patients with adherence to guidelinebased secondary prevention was 8.3\%. Compared with those without adherence, patients with adherence to guideline-based secondary prevention had lower rate of recurrent stroke (hazard ratio, 0.85 ; $95 \%$ confidence interval, 0.74 to $0.99 ; P=0.04$ ) at 12 months. Female, history of stroke, interleukin- $6 \geq 5.63 \mathrm{ng} / \mathrm{L}$, and relevant intracranial artery stenosis were independent risk factors of the residual risk.

Conclusions There was still a substantial residual risk of 12-month recurrent stroke even in patients with persistent adherence to guideline-based secondary stroke prevention. Future research should focus on efforts to reduce the residual risk.

Keywords Outcome; Secondary prevention; Risk factors; Stroke
Correspondence: Yongjun Wang Department of Neurology, Beijing Tiantan Hospital, Capital Medical University, No.119, South 4th Ring West Road, Fengtai District, Beijing 100070, China

Tel: +86-10-59975807

Fax: +86-10-59975807

E-mail:yongjunwang@ncrend.org.cn. https://orcid.org/0000-0002-99762341

Received: August 12, 2020 Revised: October 17, 2020 Accepted: October 22, 2020

\section{Introduction}

Patients with acute ischemic stroke or transient ischemic attack (TIA) suffer from approximately 10\% to 17\% risk of recurrent stroke during the first year after symptom onset. ${ }^{1,2}$ Inten- sive quality improvement in adherence to performance measures was showed to improve the prognosis of stroke. ${ }^{3}$ In recent years, there has been substantial decrease in the risk of stroke recurrence, ${ }^{4,5}$ mainly due to improvement of ischemic stroke and TIA management, especially increased adherence to guide- 
line-based secondary prevention medications (e.g., antithrombotic, antihypertensive, antidiabetic, lipid-lowing and anticoagulant treatment). ${ }^{6}$ However, the residual risk of stroke recurrence in patients with persistent adherence to guideline-based secondary stroke prevention is not yet estimated. Furthermore, it is noted that despite administration of these evidence-based therapies, substantial residual risk of stroke recurrence persists, ${ }^{7}$ indicating that the current guideline-based interventions do not adequately account for all the pathways that lead to stroke recurrence. Besides traditional vascular risk factors, there may be other risk factors (e.g., chronic inflammation) that contribute to the residual risk of stroke recurrence ${ }^{8,9}$ which might indicate the direction of efforts for stroke management in the future.

We therefore evaluated the residual risk of recurrent stroke in acute ischemic stroke or TIA with persistent adherence to guideline-based secondary stroke prevention, and identify the risk factors of the residual risk, using data from a nationwide prospective registry of patients with acute ischemic stroke and TIA in China.

\section{Methods}

\section{Study design and participants}

Data were derived from the Third China National Stroke Registry (CNSR-III), a large scale nationwide prospective registry of acute ischemic cerebrovascular events in China. ${ }^{10}$ The CNSR-III enrolled 15,166 consecutive patients with ischemic stroke and TIA from 201 hospitals in China between August 2015 and March 2018. ${ }^{10}$ The primary objectives of the CNSR-III were to establish Chinese ischemic cerebrovascular disease etiology classification based on standard diagnosis procedures and identify imaging and biological markers for prognosis of ischemic stroke. Patients with acute ischemic stroke or TIA within 7 days from symptoms onset and age older than 18 were enrolled in this study. Acute ischemic stroke was diagnosed according to the World Health Organization criteria" with confirmation by brain magnetic resonance imaging (MRI) or computed tomography (CT). The protocol of CNSR-III was approved by the ethics committee of Beijing Tiantan Hospital (KY2015001-01) and each participating site. Written informed consent was obtained from each participant or his/her representative before data collection. Data are available to researchers on request for purposes of reproducing the results by directly contacting the corresponding author.

Among all the participating sites, 169 sites had prior experience in collecting blood samples and imaging data, and agreed to participate in both the pre-specified biomarker substudy and imaging substudy. We only included patients from these 169 sites in this analysis. Patients with missing data for low-density lipoprotein cholesterol (LDL-C), high-sensitive C-reactive protein (hsCRP), interleukin-6 (IL-6) or serum creatinine were excluded from this analysis.

\section{Baseline data collection}

Trained investigators (neurologists from participating hospitals) collected baseline data through face-to-face interviews with standard operating protocol. Baseline data included demographics, medical history (prior stroke, coronary heart disease, hypertension, diabetes mellitus, dyslipidemia, and atrial fibrillation), smoking and drinking status, prestroke modified Rankin Scale, National Institutes of Health Stroke Scale (NIHSS) score at admission, and medication use.

\section{Blood sample collection and laboratory tests}

Blood samples were collected within 24 hours of admission (median time of 55 hours after index event onset). Plasma specimens were extracted, aliquoted and transported through cold chain to the central laboratory in Beijing Tiantan Hospital and stored at $-80^{\circ} \mathrm{C}$ refrigerator until tests were performed centrally and blindly.

Total cholesterol (TC), triglycerides, high-density lipoprotein cholesterol (HDL-C), LDL-C, IL-6, lipoprotein-associated phospolipase $A_{2}\left(L p-P L A_{2}\right)$, Lp-PLA 2 activity (Lp-PLA $\left.-A\right)$, hsCRP, monocyte chemoattractant protein-1, and serum creatinine were measured centrally. Then, non-HDL-C was calculated as TC-(HDL-C), and remnant lipoprotein cholesterol, corresponding to all cholesterol not found in LDL-C and HDL-C, was calculated as TC-(HDL-C)-(LDL-C). ${ }^{12}$ Estimated glomerular filtration rate (eGFR) were calculated using the Chinese modified equations from Chronic Kidney Disease Epidemiology Collaboration (CKD-EPI) with adjusted coefficient of 1.1 for the Asian population. ${ }^{13}$ An eGFR of $<60 \mathrm{~mL} / \mathrm{min} / 1.73 \mathrm{~m}^{2}$ indicated a reduced renal dysfunction. ${ }^{14}$

\section{Imaging data collection}

Brain imaging (MRI or CT), at least one vascular assessment for intracranial and extracranial arteries, and heart examination were performed at baseline following standard protocols. ${ }^{10} \mathrm{Se}-$ quences of brain MRI examinations included T1 weighted, T2 weighted, fluid-attenuated inversion recovery, diffusion-weighted imaging (DWI) with apparent diffusion coefficient maps, and magnetic resonance angiography. Imaging data were collected in Digital Imaging and Communications in Medicine (DICOM) format on discs and sent to the image research center in Beijing Tiantan Hospital. All MRI images were read centrally by two 
readers (Z.L. and J.J.) who were blinded to each other and patients' clinical information. Disagreement was further reviewed by a third reader (X.M.) and resolved by consensus.

Acute infarction was diagnosed according to the presence of hyperintense on the DWI and further classified to single acute infarction (uninterrupted lesions visible in contiguous territories) and multiple acute infarctions (more than one lesion that was topographically distinct). ${ }^{4}$ The presence of relevant intracranial artery stenosis (ICAS) was defined as 50\% to $99 \%$ stenosis or occlusion of at least one of major intracranial artery segments relevant to the index event according to the Warfarin-Aspirin Symptomatic Intracranial Disease Trial (WASID) criteria. ${ }^{15}$ Based on patient's features combined with the results of 1 or more of aforementioned diagnostic tests, all patients were classified according to the Trial of Org 10172 in Acute Stroke Treatment (TOAST) criteria: ${ }^{16}$ large-artery atherosclerosis (LAA), cardioembolism (CE), small-artery occlusion, other determined cause and undetermined cause.

\section{Patients follow-up and outcomes}

Patients were followed up by face-to-face interview at 3 months and by telephone interview at 6 and 12 months by trained research coordinators based on a standardized interview protocol. Information collected at each follow-up included cardio-/cerebrovascular events, all causes of death and medications use. Vascular events were confirmed from the treating hospital and death was either confirmed on a death certificate from the attended hospital or the local citizen registry.

The primary outcome was a new stroke defined as an aggravated primary neurologic deficit (NIHSS score increased by 4 points or above), a new neurological deficit lasting more than 24 hours or re-hospitalization with a diagnosis of ischemic stroke, intracerebral hemorrhage, or subarachnoid hemorrhage. ${ }^{11}$ The secondary outcomes included composite vascular event (stroke, myocardial infarction, or vascular death) and all cause death.

\section{Secondary prevention care}

Medications use during hospitalization, at discharge and 3-, 6-, and 12-month follow-up were also recorded. Data regarding medications during hospitalization and at discharge were collected through medical records and data regarding medications during follow-up visits were collected by interviews between site investigators (neurologists) and patients. We used five evidence-based interventions ${ }^{17}$ to document the performance of secondary prevention care of ischemic cerebrovascular event for each patient. It includes (1) antithrombotic treatment for patients who had no contraindications; (2) statins use for pa- tients with dyslipidemia or LDL-C $\geq 100 \mathrm{mg} / \mathrm{dL}$ who had no contraindications; (3) antihypertensive agents use for patients with hypertension who had no contraindications; (4) antidiabetic agents use for patients with diabetes mellitus who had no contraindications; and (5) anticoagulant agents use for atrial fibrillation who had no contraindications. Adherence to guideline-based secondary stroke prevention was expressed as an all-or-none measure and defined as proportion of patients who persistently received all of the five-performance measures at all visits (during hospitalization, at discharge, at 3, 6, and 12 months) for which the patient was eligible. ${ }^{3}$

\section{Statistical analysis}

Administration of each and total secondary prevention care performance measures at each visit were calculated and presented as proportion. Baseline variables were presented as median with interquartile range (IQR) for continuous variables and percentages for categorical variables. Baseline variables between patients included in and excluded from this analysis, and those with and without recurrent stroke were compared by using Wilcoxon rank sum test for continuous variables, and chi-square test for categorical variables, respectively. Balance of baseline characteristics between patients with and without adherence to guideline-based secondary stroke prevention was checked using the absolute standardized difference. An absolute standardized difference greater than 0.10 is approximately equivalent to $P<0.05$ and indicates significant imbalance of a baseline covariate between groups. ${ }^{18}$

The associations between adherence to guideline-based secondary stroke prevention and outcomes were assessed using usual univariable and multivariable Cox proportional hazards model for outcomes of stroke, composite vascular event and death. Age, sex, and all the covariates with an absolute standardized difference greater than 0.10 in the univariable analyses were adjusted in the multivariable models, including hypertension, diabetes, atrial fibrillation, TC, LDL-C, non-HDL-C, and stroke etiology. The proportional hazards assumption for the Cox model was tested by adding a time-dependent covariate with interaction of group and a logarithmic function of survival time in the model. We further used the Fine and Gray regression model for a subdistribution function treating non-stroke/nonvascular death as a competing event. ${ }^{19}$ In sensitivity analysis, the associations were further assessed using a propensity score approach with inverse probability of treatment weighted estimation. The propensity score for adherence to guideline-based secondary prevention was generated for each patient regardless of the outcome by using a nonparsimonious multivariable logistic regression model with all covariates in Table 1 included in 


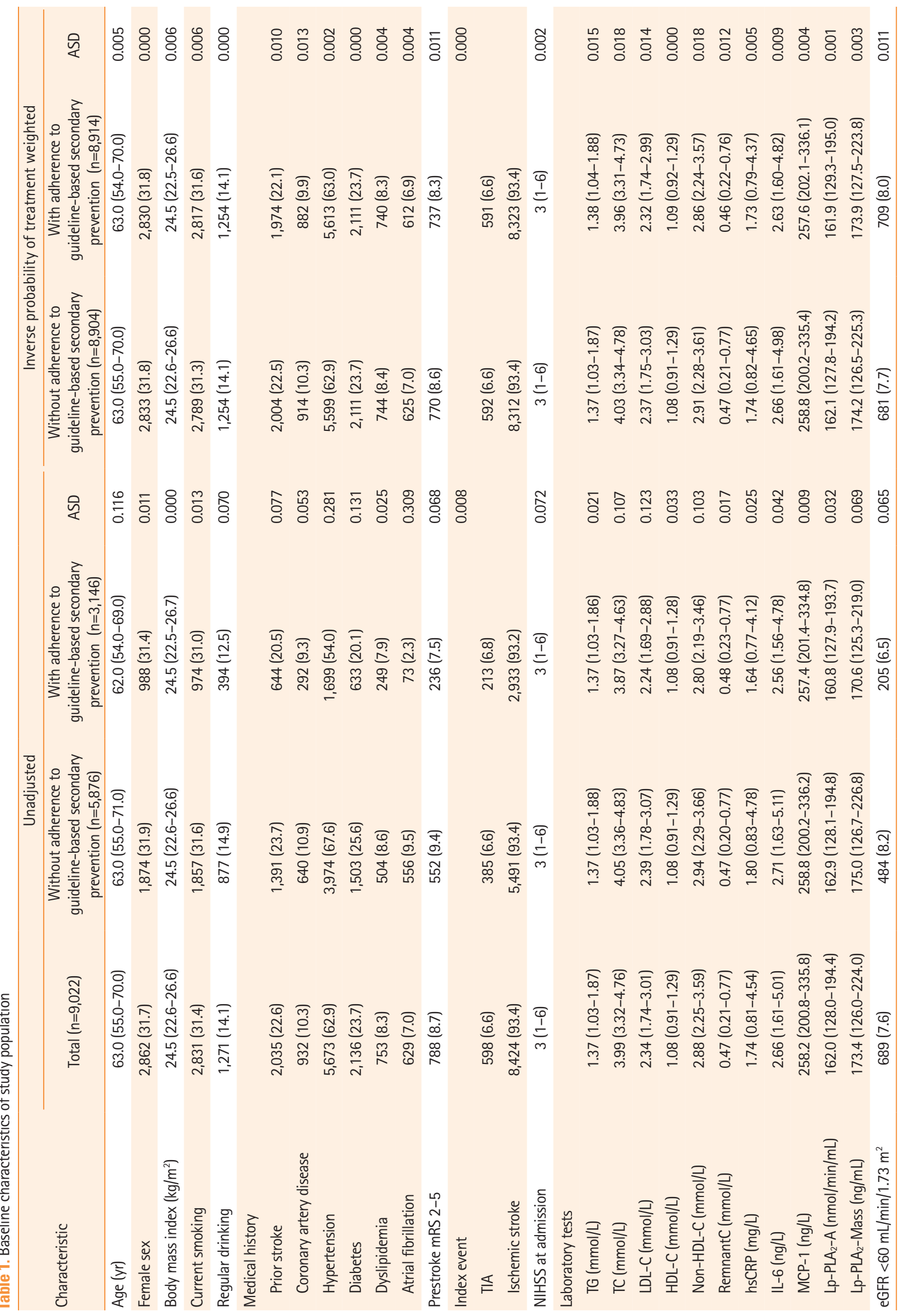




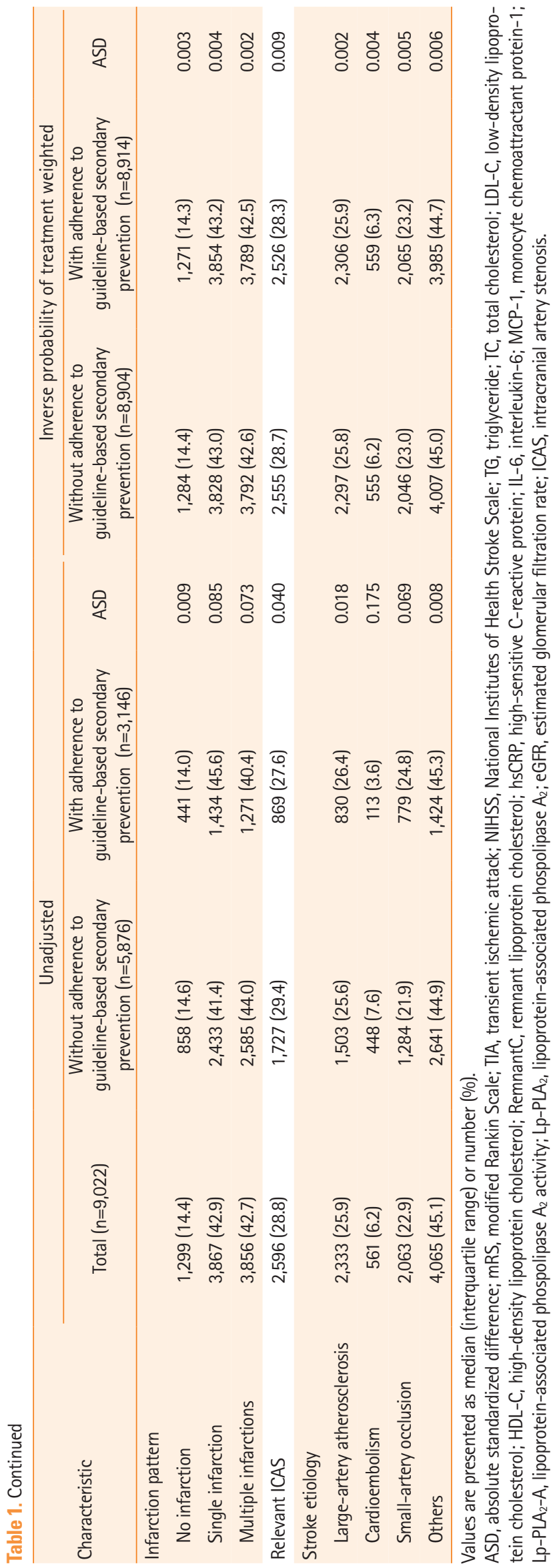

the model. Inverse probability of treatment weighted estimation was then defined as the inverse of the estimated propensity score for patients with adherence to guideline-based secondary stroke prevention and the inverse of one minus the estimated propensity score for those without adherence to guidelinebased secondary stroke prevention. ${ }^{20}$ Hazard ratios (HRs) with their 95\% confidence intervals (Cls) were calculated.

Risks of recurrent stroke at 12 months were calculated in total population (indicating risk under current clinical practice) and those with adherence to guideline-based secondary stroke prevention (indicating residual risk under adherence to guideline-based secondary stroke prevention) in all patients and each etiological subtype, respectively. Receiver operating characteristic (ROC) cure analysis was used to identify the cut-off point of IL-6 for recurrent stroke. Independent risk factors of residual risk for 12-month recurrent stroke were determined using multivariable Cox regression in patients with adherence to guideline-based standard secondary prevention. All the covariates with $P<0.05$ in the univariable analysis were included in the model and selected with stepwise method. Based on the results of multivariable regression, residual risk of recurrent stroke was further estimated in a hypothetical scenario with anti-inflammatory therapy and treatment for ICAS in population with adherence to guideline-based secondary stroke prevention using the data of population without these two risk factors. We presented the time to first new stroke in different scenarios by the Kaplan-Meier curves.

To assess the contribution of each risk factor to the residual risk of new stroke, attributable risk percent (ARP) (1) and population attributable risk percent (PARP) (2) were calculated as follows: $:^{21}$

$$
\begin{aligned}
& \mathrm{ARP}=\left(\mathrm{I}_{\mathrm{e}}-\mathrm{I}_{0}\right) / \mathrm{l}_{\mathrm{e}} \times 100 \% \mathrm{o}_{\mathrm{t}}(1) \\
& \mathrm{PARP}=\left(\mathrm{I}_{\mathrm{t}}-\mathrm{I}_{\mathrm{o}}\right) / \mathrm{I}_{\mathrm{t}} \times 100 \%, \mathrm{o}_{\mathrm{t}}(2)
\end{aligned}
$$

$I_{t}$ denotes rate of new stroke in the total population; $I_{0}$ is rate of new stroke in population without the risk factor; $l_{e}$ is rate of new stroke in population with the risk factor.

All analyses were conducted with SAS software version 9.4 (SAS Institute Inc, Cary, NC, USA). P-values less than 0.05 were considered statistically significant and all tests were 2 -sided.

\section{Results}

\section{Study participants and characteristics}

A total of 9,733 patients with ischemic stroke or TIA in 169 sites participated in both the biomarker and imaging substudies. After further exclusion of 711 patients with missing data of laboratory tests or lost to follow-up at 12 months, a total of 9,022 patients with ischemic stroke or TIA were included in 
15,166 Ischemic stroke or TIA patients in 201 sites in CNSR-III

5,433 Patients in 32 sites excluded

3,905 Not included in biomarker substudy

2,154 Not included in imaging substudy

9,733 Patients in 169 sites having blood sample and MRI

111 Patients excluded

$65(0.7 \%)$ With missing data of LDL-C

315 (3.2\%) With missing data of hsCRP

$191(2.0 \%)$ With missing data of IL-6

$8(0.1 \%)$ With missing data of serum creatinine

$227(2.3 \%)$ Lost to follow-up at 12 months

9,022 Ischemic stroke or TIA patients in this analysis

Figure 1. Flow chart of study population. TIA, transient ischemic attack; CNSR-III, Third China National Stroke Registry; MRI, magnetic resonance imaging; LDL-C, low-density lipoprotein cholesterol; hsCRP, high-sensitive C-reactive protein; IL-6, interleukin-6.

this analysis (Figure 1). Patients included in and those excluded from this analysis were largely comparable (Supplementary Table 1). Among these patients, 2,862 (31.7\%) were female and the median age was 63.0 years (IOR, 55.0 to 70.0); 8,424 (93.4\%) patients had an index event of ischemic stroke and 598 (6.6\%) patients had TIA (Table 1). The median of NIHSS score at admission was 3 (IQR, 1 to 6).

\section{Administration of secondary prevention medication}

Among all the eligible patients, there were 97.4\%, 96.8\%, $80.4 \%, 60.4 \%$, and $28.6 \%$ patients received the antithrombotic treatment, statin, antidiabetic, antihypertensive, and anticoagulant agents during hospitalization, respectively (Supplementary Figure 1). Administration of these secondary prevention medications at discharge and at 3, 6, and 12 months were almost similar to that during hospitalization, but a little lower for antithrombotic treatment, statin, and antidiabetic treatment and a little higher for antihypertensive and anticoagulant treatment (Supplementary Figure 1). There were 61.0\%, 62.3\%, $59.3 \%, 58.1 \%$, and $54.4 \%$ patients adherent to all the five performance measures at each visit (during hospitalization, at discharge, at 3,6, and 12 months), respectively.

Among all patients, there were 3,146 (34.9\%) patients with adherence to guideline-based secondary stroke prevention (received all the five performance measures at all visits). Compared with those without adherence, patients with adherence to guideline-based secondary stroke prevention were younger, less likely to have medical histories (hypertension, diabetes, atrial fibrillation) and CE etiological subtype, had lower level of TC, LDL-C, and non-HDL-C (Table 1).

\section{Residual risk of recurrent stroke and risk factors} Among all the 9,022 patients, 864 (9.6\%) and 912 (10.1\%) patients had recurrent stroke and composite vascular event at 12 months, respectively. Compared with those without adherence, patients with adherence to guideline-based secondary stroke prevention had lower rate of new stroke (8.3\% vs. 10.2\%; adjusted hazard ratio [aHR], $0.85 ; 95 \% \mathrm{Cl}, 0.74$ to $0.99 ; P=0.04)$ and composite vascular event (8.7\% vs. $10.9 \%$; aHR, $0.85 ; 95 \% \mathrm{Cl}, 0.74$ to $0.98 ; P=0.03$ ) at 12 months (Table 2 and Figure 2). All of the proportional hazard assumptions were met $(P=0.16$ for new stroke and $P=0.10$ for composite vascular event). Similar results were observed using competing-risks models and propensity score approaches with inverse probability of treatment weighted estimation (Table 2). Further analysis showed that adherence to guideline-based secondary prevention care mainly reduced the residual risks in LAA subtype (Supplementary Table 2).

Among patients with adherence to guideline-based secondary stroke prevention, those with recurrent stroke at 12 months were more likely to be female, have history of stroke and diabetes, multiple infarctions, relevant ICAS and LAA etiological subtype, higher level of hsCRP and IL-6, but less likely to be current smoking (Supplementary Table 3). In the multivariable regression model, female, history of stroke, higher IL-6 level, and relevant ICAS were independent risk factors of residual risk of 12-month new stroke (Table 3 ).

Higher IL-6 level (defined by cut-off values of $\geq 5.63 \mathrm{ng} / \mathrm{L}$ derived from the ROC analysis) and relevant ICAS attributed to $11.2 \%$ and $14.6 \%$ of the total population residual risk respectively, and together contributed to $17.6 \%$. Residual risk of 12-month recurrent stroke was estimated to be 6.9\% in hypothetical scenario with further anti-inflammatory therapy and treatment for ICAS estimated using data of subgroup patients without these two risk factors in population with adherence to guideline-based secondary stroke prevention (Figure 2 and Supplementary Table 2).

\section{Discussion}

In the nationwide, multicenter, prospective registry in China, we found that although substantially reduced by secondary prevention treatment, there was still 8.3\% residual risk of 12-month recurrent stroke even in patients with persistent adherence to guideline-based secondary stroke prevention. Female, history of stroke, higher IL-6 level, and relevant ICAS 


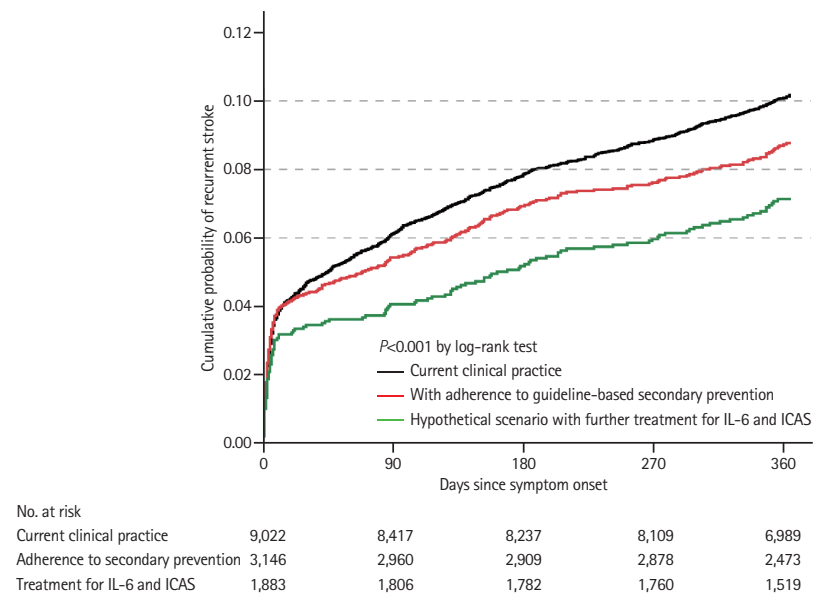

Figure 2. Residual risk of recurrent stroke in different scenarios. IL-6, interleukin-6; ICAS, intracranial artery stenosis. were independent risk factors of the residual risk. Hypothetical anti-inflammatory therapy and treatment for ICAS may be associated with further reduction of approximately $17 \%$ of the residual risk in population with adherence to guideline-based secondary stroke prevention.

The residual risk of recurrent stroke in patients under guideline-based secondary prevention management has not been well-estimated and varied ranging from approximately $6 \%$ to $14 \%$ at 12 months. $^{7,22,23}$ Differences in patient characteristics, severity of stroke, intensity of secondary prevention management, different ethnicities, and setting of studies may contribute to the heterogeneity of these estimates. Our study observed 8.3\% residual risk of 12-month recurrent stroke despite administration of these evidence-based therapies in a nationwide, prospective registry in China. Future research may focus on

Table 2. Outcomes at 12 months in patients with or without adherence to guideline-based secondary prevention

\begin{tabular}{|c|c|c|c|c|c|}
\hline $\begin{array}{l}\text { Outcomes at } \\
3 \text { months }\end{array}$ & Model & $\begin{array}{c}\text { Without adherence to } \\
\text { guideline-based secondary } \\
\text { prevention }\end{array}$ & $\begin{array}{c}\text { With adherence to } \\
\text { guideline-based secondary } \\
\text { prevention }\end{array}$ & $\mathrm{HR}(95 \% \mathrm{Cl})$ & $P$ \\
\hline \multirow[t]{6}{*}{ Stroke } & Univariable Cox regression & $602(10.2)$ & $262(8.3)$ & $0.81(0.70-0.93)$ & 0.003 \\
\hline & Multivariable Cox regression* & & & $0.85(0.74-0.99)$ & 0.037 \\
\hline & IPTW Cox regression & & & $0.84(0.76-0.92)$ & $<0.001$ \\
\hline & Univariable competing-risks model & & & $0.80(0.68-0.93)$ & 0.004 \\
\hline & Multivariable competing-risks model ${ }^{*}$ & & & $0.85(0.72-0.99)$ & 0.036 \\
\hline & IPTW competing-risks model & & & $0.80(0.68-0.93)$ & 0.004 \\
\hline \multirow{6}{*}{$\begin{array}{l}\text { Composite vascular } \\
\text { event }^{+}\end{array}$} & Univariable Cox regression & 639 (10.9) & $273(8.7)$ & $0.79(0.69-0.91)$ & 0.001 \\
\hline & Multivariable Cox regression* & & & $0.85(0.74-0.98)$ & 0.027 \\
\hline & IPTW Cox regression & & & $0.83(0.75-0.91)$ & $<0.001$ \\
\hline & Univariable competing-risks model & & & $0.76(0.66-0.88)$ & $<0.001$ \\
\hline & Multivariable competing-risks model ${ }^{*}$ & & & $0.82(0.71-0.95)$ & 0.010 \\
\hline & IPTW competing-risks model & & & $0.76(0.66-0.89)$ & $<0.001$ \\
\hline \multirow[t]{3}{*}{ Death } & Univariable Cox regression & $224(3.8)$ & $68(2.2)$ & $0.56(0.43-0.74)$ & $<0.001$ \\
\hline & Multivariable Cox regression & & & $0.69(0.52-0.91)$ & 0.009 \\
\hline & IPTW Cox regression & & & $0.66(0.55-0.78)$ & $<0.001$ \\
\hline
\end{tabular}

Values are presented as number (\%).

$\mathrm{HR}$, hazard ratio; $\mathrm{Cl}$, confidence interval; IPTW, inverse probability of treatment weighted.

*Adjusted for age, sex, hypertension, diabetes, atrial fibrillation, triglyceride, low-density lipoprotein cholesterol, non-high-density lipoprotein cholesterol, and stroke etiology; ${ }^{+}$Composite vascular event was defined as a new stroke, myocardial infarction or vascular death.

Table 3. Independent risk factors of residual risk of 12-month recurrent stroke

\begin{tabular}{lccccccc}
\hline Characteristic & aHR $(95 \% \mathrm{Cl})$ & $P$ & $\mathrm{I}_{\mathrm{t}}(\%)$ & $\mathrm{I}_{\mathrm{o}}\left(\mathrm{n}_{\mathrm{o}} / \mathrm{N}_{\mathrm{o}}\right)$ & $\mathrm{I}_{\mathrm{e}}\left(\mathrm{n}_{\mathrm{e}} / \mathrm{N}_{\mathrm{e}}\right)$ & ARP $(\%)$ & PARP $(\%)$ \\
\hline Female sex & $1.46(1.14-1.87)$ & 0.003 & 8.33 & $7.32(158 / 2,158)$ & $10.53(104 / 988)$ & 30.5 & 12.1 \\
History of stroke & $1.39(1.06-1.83)$ & 0.019 & 8.33 & $7.71(193 / 2,502)$ & $10.71(69 / 644)$ & 28.0 & 7.4 \\
IL-6 $\geq 5.63 \mathrm{ng} / \mathrm{L}$ & $1.65(1.26-2.16)$ & $<0.001$ & 8.33 & $7.40(186 / 2,515)$ & $12.04(76 / 631)$ & 38.5 & 11.2 \\
Relevant ICAS & $1.57(1.22-2.02)$ & $<0.001$ & 8.33 & $7.11(162 / 2,277)$ & $11.51(100 / 869)$ & 38.2 & 14.6 \\
\hline
\end{tabular}

aHR, adjusted hazard ratio; $\mathrm{Cl}$, confidence interval; $I_{t}$, rate of new stroke in the total population; $I_{0}$, rate of new stroke in population without the risk factor; $I_{\mathrm{e}}$ rate of new stroke in population with the risk factor; ARP, attributable risk percent; PARP, population attributable risk percent; IL-6, interleukin-6; ICAS, intracranial artery stenosis. 
identification of non-traditional risk factors and specific effective strategies to further reduce the residual risk in patents under guideline-based secondary prevention management.

Recent studies demonstrated that chronic inflammation was an independent predictor of vascular events both in primary and secondary prevention setting. ${ }^{24-27}$ In population-based studies, elevated IL-6 was found to be associated with subclinical atherosclerosis and incident stroke. ${ }^{24,25}$ Other previous studies demonstrated that patients with elevated hsCRP had an increased risk of recurrent stroke. ${ }^{26,27}$ Although anti-inflammatory therapy with canakinumab can reduce the rate of recurrent cardiovascular events in patients with myocardial infarction in the Canakinumab Anti-inflammatory Thrombosis Outcome Study (CANTOS) trial, ${ }^{28}$ well-designed randomized trial of anti-inflammatory therapy in patients with ischemic stroke has not yet been performed. Our study showed that inflammation was an independent risk factor of recurrent stroke even in patients under guideline-based secondary prevention medications, emphasizing that anti-inflammatory therapy might be considered in secondary prevention clinical practice. Therefore, randomized trials estimating the effect of anti-inflammatory therapy in patients with ischemic stroke should be performed in future.

A previous study showed a high prevalence (46.6\%; relevant or not) of ICAS in patients with cerebrovascular disease and it was an independent predictor for recurrent stroke. ${ }^{29}$ Despite its heavy burden, there is no specific treatment for ICAS. The Stenting and Aggressive Medical Management for Preventing Recurrent stroke in Intracranial Stenosis (SAMMPRIS) trial demonstrated benefit of aggressive medical management over stenting in high-risk patients with ICAS. ${ }^{30}$ Our study showed that even with persistent adherence to guideline-based secondary prevention medications, relevant ICAS was still an important independent risk factor of the residual risk, indicating that more aggressive intervention should be developed to treat this risk factor.

This study has several limitations. First, although secondary prevention medications were administrated in each visit, whether secondary prevention goals (blood pressure, glucose, and lipoprotein lowing, etc.) were achieved was not assessed, thus leading to a potential misclassification of patients. Insufficient control of the risk factors in the real world may lead to overestimate the residual risk. Second, patients with adherence to guideline-based secondary stroke prevention had less vascular risk factors than those without adherence, which may cause underestimate the residual risk. Third, residual risk in hypothetical scenario with treatment for inflammation and ICAS was estimated using the data of population without these two risk factors. This may be different from the real effect of the treatments. Fourth, high level of inflammatory markers like hsCRP and IL-6 may increase due to stress response to the acute events and not reflect chronic inflammation. Fifth, medications during follow-up visits were collected by interviews between neurologists and patients. Patients' false recall and their little understanding of the medication may attenuate recorded information's validity. Finally, the external generalizability of our findings is limited as participants were restricted to Chinese patients. Selection bias may also exist as the low proportion of atrial fibrillation (7.0\% in CNSR-III vs. $9.6 \%$ in CNSR-II') and rate of death (3.2\% in CNSR-III vs. $8.4 \%$ in CNSR- $-I^{31}$ ) were observed in this population. Further validation was required in other populations or settings.

\section{Conclusions}

In patients with ischemic stroke or TIA and persistent adherence to guideline-based secondary stroke prevention, there was still a substantial residual risk of 12-month recurrent stroke. Female, history of stroke, inflammation, and relevant ICAS were independent risk factors of the residual risk. Future research should focus on efforts to reduce the residual risk targeting these non-traditional risk factors.

\section{Supplementary materials}

Supplementary materials related to this article can be found online at https://doi.org/10.5853/jos.2020.03391.

\section{Disclosure}

The authors have no financial conflicts of interest.

\section{Acknowledgments}

This study is supported by grants from the National Science and Technology Major Project (2017ZX09304018), National Key R\&D Program of China (2018YFC1312903, 2017YFC1310902, 2018YFC1311700 and 2018YFC1311706), National Natural Science Foundation of China (81971091), Beijing Hospitals Authority Youth Programme (OML20190501), Beijing Municipal Science \& Technology Commission (D171100003017002, Z18110000 1818001).

\section{References}

1. Chen Y, Wright N, Guo Y, Turnbull I, Kartsonaki C, Yang L, et 
al. Mortality and recurrent vascular events after first incident stroke: a 9-year community-based study of 0.5 million Chinese adults. Lancet Glob Health 2020;8:e580-e590.

2. Lin B, Zhang Z, Mei Y, Wang $C, X u H$, Liu L, et al. Cumulative risk of stroke recurrence over the last 10 years: a systematic review and meta-analysis. Neurol Sci 2020 Oct 10 [Epub]. https://doi.org/10.1007/s10072-020-04797-5.

3. Wang Y, Li Z, Zhao X, Wang C, Wang X, Wang D, et al. Effect of a multifaceted quality improvement intervention on hospital personnel adherence to performance measures in patients with acute ischemic stroke in China: a randomized clinical trial. JAMA 2018;320:245-254.

4. Amarenco P, Lavallée PC, Labreuche J, Albers GW, Bornstein NM, Canhão $P$, et al. One-year risk of stroke after transient ischemic attack or minor stroke. N Engl J Med 2016;374: 1533-1542.

5. Xie $X$, Wang $X$, Li Z, Zhao X, Miao Z, Liu L, et al. Prognostic value of international normalized ratio in ischemic stroke patients without atrial fibrillation or anticoagulation therapy. J Atheroscler Thromb 2019;26:378-387.

6. Li Z, Wang C, Zhao X, Liu L, Wang C, Li H, et al. Substantial progress yet significant opportunity for improvement in stroke care in China. Stroke 2016;47:2843-2849.

7. Ji R, Liu G, Shen $H$, Wang Y, Li H, Peterson E, et al. Persistence of secondary prevention medications after acute ischemic stroke or transient ischemic attack in Chinese population: data from China National Stroke Registry. Neurol Res 2013; 35:29-36.

8. Patel KV, Pandey A, de Lemos JA. Conceptual framework for addressing residual atherosclerotic cardiovascular disease risk in the era of precision medicine. Circulation 2018;137: 2551-2553.

9. Ridker PM. Residual inflammatory risk: addressing the obverse side of the atherosclerosis prevention coin. Eur Heart $J$ 2016;37:1720-1722.

10. Wang $Y$, Jing J, Meng $X$, Pan $Y$, Wang $Y$, Zhao $X$, et al. The Third China National Stroke Registry (CNSR-III) for patients with acute ischaemic stroke or transient ischaemic attack: design, rationale and baseline patient characteristics. Stroke Vasc Neurol 2019;4:158-164.

11. Stroke: 1989. Recommendations on stroke prevention, diagnosis, and therapy. Report of the WHO Task Force on Stroke and other Cerebrovascular Disorders. Stroke 1989;20:14071431.

12. Nordestgaard BG, Langlois MR, Langsted A, Chapman MJ, Aakre KM, Baum $\mathrm{H}$, et al. Quantifying atherogenic lipoproteins for lipid-lowering strategies: consensus-based recommendations from EAS and EFLM. Atherosclerosis 2020;
294:46-61.

13. Ma YC, Zuo L, Chen JH, Luo Q, Yu XQ, Li Y, et al. Modified glomerular filtration rate estimating equation for Chinese patients with chronic kidney disease. J Am Soc Nephrol 2006; 17:2937-2944.

14. Levey AS, Coresh J, Greene T, Stevens LA, Zhang YL, Hendriksen $S$, et al. Using standardized serum creatinine values in the modification of diet in renal disease study equation for estimating glomerular filtration rate. Ann Intern Med 2006; 145:247-254.

15. Samuels OB, Joseph GJ, Lynn MJ, Smith HA, Chimowitz MI. A standardized method for measuring intracranial arterial stenosis. AJNR Am J Neuroradiol 2000;21:643-646.

16. Adams HP Jr, Bendixen BH, Kappelle $\sqcup$, Biller J, Love BB, Gordon DL, et al. Classification of subtype of acute ischemic stroke. Definitions for use in a multicenter clinical trial. TOAST. Trial of Org 10172 in Acute Stroke Treatment. Stroke 1993;24:35-41.

17. Pan Y, Chen R, Li Z, Li H, Zhao X, Liu L, et al. Socioeconomic status and the quality of acute stroke care: The China $\mathrm{Na}-$ tional Stroke Registry. Stroke 2016;47:2836-2842.

18. Xian $Y$, Holloway RG, Chan PS, Noyes $K$, Shah MN, Ting HH, et al. Association between stroke center hospitalization for acute ischemic stroke and mortality. JAMA 2011;305:373380.

19. Fine JP, Gray RJ. A proportional hazards model for the subdistribution of a competing risk. J Am Stat Assoc 1999;94: 496-509.

20. Brookhart MA, Wyss R, Layton JB, Stürmer T. Propensity score methods for confounding control in nonexperimental research. Circ Cardiovasc Qual Outcomes 2013;6:604-611.

21. Jiang J, Cai $X$, Pan $Y$, Du $X$, Zhu H, Yang $X$, et al. Relationship of obesity to adipose tissue insulin resistance. BMJ Open Diabetes Res Care 2020;8:e000741.

22. Bushnell CD, Olson DM, Zhao X, Pan W, Zimmer LO, Goldstein $\mathrm{LB}$, et al. Secondary preventive medication persistence and adherence 1 year after stroke. Neurology 2011;77:1182-1190.

23. Chimowitz MI, Lynn MJ, Derdeyn CP, Turan TN, Fiorella D, Lane $B F$, et al. Stenting versus aggressive medical therapy for intracranial arterial stenosis. N Engl J Med 2011;365:9931003.

24. Cainzos-Achirica M, Enjuanes $C$, Greenland P, McEvoy JW, Cushman $M$, Dardari $Z$, et al. The prognostic value of interleukin 6 in multiple chronic diseases and all-cause death: the Multi-Ethnic Study of Atherosclerosis (MESA). Atherosclerosis 2018;278:217-225.

25. Jenny NS, Callas PW, Judd SE, McClure LA, Kissela B, Zakai $N A$, et al. Inflammatory cytokines and ischemic stroke risk: 
the REGARDS cohort. Neurology 2019;92:e2375-e2384.

26. Elkind MS, Luna JM, McClure LA, Zhang Y, Coffey CS, Roldan $A$, et al. C-reactive protein as a prognostic marker after lacunar stroke: levels of inflammatory markers in the treatment of stroke study. Stroke 2014;45:707-716.

27. Li J, Zhao X, Meng X, Lin J, Liu L, Wang C, et al. High-sensitive c-reactive protein predicts recurrent stroke and poor functional outcome: subanalysis of the clopidogrel in highrisk patients with acute nondisabling cerebrovascular events trial. Stroke 2016;47:2025-2030.

28. Ridker PM, Everett BM, Thuren T, MacFadyen JG, Chang WH, Ballantyne $C_{\text {, et }}$ al. Antiinflammatory therapy with canakinumab for atherosclerotic disease. N Engl J Med 2017;377: 1119-1131.
29. Wang $Y$, Zhao $X$, Liu L, Soo YO, Pu Y, Pan Y, et al. Prevalence and outcomes of symptomatic intracranial large artery stenoses and occlusions in China: the Chinese Intracranial Atherosclerosis (CICAS) Study. Stroke 2014;45:663-669.

30. Derdeyn CP, Chimowitz MI, Lynn MJ, Fiorella D, Turan TN, Janis LS, et al. Aggressive medical treatment with or without stenting in high-risk patients with intracranial artery stenosis (SAMMPRIS): the final results of a randomised trial. Lancet 2014;383:333-341.

31. Yang XM, Rao ZZ, Gu HQ, Zhao XQ, Wang CJ, Liu LP, et al. Atrial fibrillation known before or detected after stroke share similar risk of ischemic stroke recurrence and death. Stroke 2019;50:1124-1129. 
Supplementary Table 1. Baseline characteristics of patients included in and excluded from this analysis

\begin{tabular}{|c|c|c|c|}
\hline Characteristic & Excluded $(n=711)$ & Included $(n=9,022)$ & $P$ \\
\hline Age (yr) & $62.0(54.0-70.0)$ & $63.0(55.0-70.0)$ & 0.538 \\
\hline Female sex & $195(27.4)$ & $2,862(31.7)$ & 0.018 \\
\hline Body mass index $\left(\mathrm{kg} / \mathrm{m}^{2}\right)$ & $24.5(22.6-26.4)$ & $24.5(22.6-26.6)$ & 0.720 \\
\hline Current smoking & $257(36.1)$ & $2,831(31.4)$ & 0.009 \\
\hline Regular drinking & 112 (15.8) & $1,271(14.1)$ & 0.221 \\
\hline \multicolumn{4}{|l|}{ Medical history } \\
\hline Prior stroke & 128 (18.0) & $2,035(22.6)$ & 0.005 \\
\hline Coronary artery disease & $84(11.8)$ & $932(10.3)$ & 0.213 \\
\hline Hypertension & $453(63.7)$ & $5,673(62.9)$ & 0.658 \\
\hline Diabetes & $153(21.5)$ & $2,136(23.7)$ & 0.192 \\
\hline Dyslipidemia & $54(7.6)$ & 753 (8.3) & 0.484 \\
\hline Atrial fibrillation & $50(7.0)$ & $629(7.0)$ & 0.951 \\
\hline Prestroke mRS 2-5 & $67(9.4)$ & $788(8.7)$ & 0.532 \\
\hline Index event & & & 0.139 \\
\hline TIA & $37(5.2)$ & $598(6.6)$ & \\
\hline Ischemic stroke & $674(94.8)$ & $8,424(93.4)$ & \\
\hline NIHSS at admission & $3.0(2.0-6.0)$ & $3.0(1.0-6.0)$ & 0.003 \\
\hline Stroke etiology & & & 0.432 \\
\hline Large-artery atherosclerosis & $204(28.7)$ & $2,333(25.9)$ & \\
\hline Cardioembolism & $43(6.0)$ & $561(6.2)$ & \\
\hline Small-artery occlusion & $156(21.9)$ & $2,063(22.9)$ & \\
\hline Others & 308 (43.3) & $4,065(45.1)$ & \\
\hline
\end{tabular}

Values are presented as median (interquartile range) or number (\%).

mRS, modified Rankin Scale; TIA, transient ischemic attack; NIHSS, National Institutes of Health Stroke Scale.

Supplementary Table 2. Risk for 12-month recurrent stroke according to etiological subtypes

\begin{tabular}{|c|c|c|c|c|c|}
\hline Scenarios & Total & LAA & CE & SAO & Others \\
\hline Current clinical practice & $864 / 9,022(9.6)$ & $290 / 2,333(12.4)$ & $68 / 561(12.1)$ & $143 / 2,063(6.9)$ & $363 / 4,065$ (8.9) \\
\hline $\begin{array}{l}\text { With adherence to guideline-based secondary stroke } \\
\text { prevention }\end{array}$ & $262 / 3,146(8.3)$ & $92 / 830(11.1)$ & $14 / 113(12.4)$ & $47 / 779(6.0)$ & $109 / 1,424(7.7)$ \\
\hline $\begin{array}{l}\text { Without adherence to guideline-based secondary stroke } \\
\text { prevention }\end{array}$ & $602 / 5,876(10.2)$ & $198 / 1,503(13.2)$ & $54 / 448$ (12.1) & $96 / 1,284(7.5)$ & $254 / 2,641$ (9.6) \\
\hline $\begin{array}{l}\text { Hypothetical scenario with further anti-inflammatory } \\
\text { therapy and treatment for ICAS }\end{array}$ & $128 / 1,866(6.9)$ & - & $11 / 77(14.3)$ & 36/662 (5.4) & $81 / 1,127(7.2)$ \\
\hline
\end{tabular}

Values are presented as number (\%).

LAA, large-artery atherosclerosis; $\mathrm{CE}$, cardioembolism; $\mathrm{SAO}$, small-artery occlusion; ICAS, intracranial artery stenosis. 
Supplementary Table 3. Baseline characteristics of patients with adherence to guideline-based secondary stroke prevention according to 12-month recurrent stroke or not

\begin{tabular}{|c|c|c|c|}
\hline Characteristic & $\begin{array}{l}\text { Recurrent stroke } \\
\qquad(n=262)\end{array}$ & $\begin{array}{l}\text { Non-recurrent stroke } \\
\qquad(n=2,884)\end{array}$ & $P$ \\
\hline Age (yr) & $63.0(55.0-71.0)$ & $62.0(53.0-69.0)$ & 0.087 \\
\hline Female sex & $104(39.7)$ & $884(30.7)$ & 0.003 \\
\hline Body mass index $\left(\mathrm{kg} / \mathrm{m}^{2}\right)$ & $24.7(22.5-27.0)$ & $24.5(22.5-26.7)$ & 0.493 \\
\hline Current smoking & $59(22.5)$ & $915(31.7)$ & 0.002 \\
\hline Regular drinking & $33(12.6)$ & $361(12.5)$ & 0.971 \\
\hline \multicolumn{4}{|l|}{ Medical history } \\
\hline Prior stroke & $69(26.3)$ & $575(19.9)$ & 0.014 \\
\hline Coronary artery disease & $27(10.3)$ & $265(9.2)$ & 0.551 \\
\hline Hypertension & 145 (55.3) & $1,554(53.9)$ & 0.650 \\
\hline Diabetes & $66(25.2)$ & $567(19.7)$ & 0.033 \\
\hline Dyslipidemia & $23(8.8)$ & $226(7.8)$ & 0.590 \\
\hline Atrial fibrillation & $5(1.9)$ & $68(2.4)$ & 0.644 \\
\hline Prestroke mRS 2-5 & $27(10.3)$ & $209(7.2)$ & 0.072 \\
\hline Index event & & & 0.047 \\
\hline TIA & $10(3.8)$ & $203(7.0)$ & \\
\hline Ischemic stroke & $252(96.2)$ & $2,681(93.0)$ & \\
\hline NIHSS at admission & $3(2-6)$ & $3(1-6)$ & 0.304 \\
\hline \multicolumn{4}{|l|}{ Laboratory tests } \\
\hline TG $(\mathrm{mmol} / \mathrm{L})$ & $1.28(0.97-1.78)$ & $1.38(1.04-1.86)$ & 0.058 \\
\hline $\mathrm{TC}(\mathrm{mmol} / \mathrm{L})$ & $3.88(3.15-4.59)$ & $3.87(3.28-4.64)$ & 0.544 \\
\hline $\mathrm{LDL}-\mathrm{C}(\mathrm{mmol} / \mathrm{L})$ & $2.19(1.65-2.85)$ & $2.25(1.69-2.88)$ & 0.476 \\
\hline HDL-C (mmol/L) & $1.08(0.90-1.26)$ & $1.08(0.91-1.28)$ & 0.626 \\
\hline Non-HDL-C (mmol/L) & $2.82(2.11-3.43)$ & $2.80(2.20-3.46)$ & 0.674 \\
\hline RemnantC (mmol/L) & $0.46(0.24-0.73)$ & $0.48(0.23-0.77)$ & 0.639 \\
\hline hsCRP (mg/L) & $2.22(0.94-5.77)$ & $1.57(0.76-3.99)$ & $<0.001$ \\
\hline IL-6 (ng/L) & $2.96(1.75-6.14)$ & $2.52(1.56-4.67)$ & 0.001 \\
\hline MCP-1 (ng/L) & $259.1(201.1-349.0)$ & $257.3(201.9-334.2)$ & 0.992 \\
\hline Lp-PLA2-A (nmol/min/mL) & 163.6 (130.6-199.2) & $160.4(127.6-193.1)$ & 0.303 \\
\hline Lp-PLA2-Mass (ng/mL) & 172.6 (122.9-218.8) & 170.5 (125.4-219.0) & 0.994 \\
\hline $\mathrm{eGFR}<60 \mathrm{~mL} / \mathrm{min} / 1.73 \mathrm{~m}^{2}$ & $21(8.0)$ & $184(6.4)$ & 0.305 \\
\hline Infarction pattern & & 0.02 & \\
\hline No infarction & $28(10.7)$ & $413(14.3)$ & \\
\hline Single infarction & $108(41.2)$ & $1,326(46.0)$ & \\
\hline Multiple infarctions & $126(48.1)$ & $1,145(39.7)$ & \\
\hline Relevant ICAS & $100(38.2)$ & 769 (26.7) & $<0.001$ \\
\hline Stroke etiology & & & $<0.001$ \\
\hline Large-artery atherosclerosis & $92(35.1)$ & $738(25.6)$ & \\
\hline Cardioembolism & $14(5.3)$ & $99(3.4)$ & \\
\hline Small-artery occlusion & $47(17.9)$ & $732(25.4)$ & \\
\hline Others & $109(41.6)$ & $1,315(45.6)$ & \\
\hline
\end{tabular}

Values are presented as median (interquartile range) or number (\%).

mRS, modified Rankin Scale; TIA, transient ischemic attack; NIHSS, National Institutes of Health Stroke Scale; TG, triglyceride; TC, total cholesterol; LDL-C, low-density lipoprotein cholesterol; HDL-C, high-density lipoprotein cholesterol; RemnantC, remnant lipoprotein cholesterol; hsCRP, high-sensitive C-reactive protein; IL-6, interleukin-6; MCP-1, monocyte chemoattractant protein-1; Lp-PLA2-A, lipoprotein-associated phospolipase A2 activity; Lp-PLA2, lipoproteinassociated phospolipase A2; eGFR, estimated glomerular filtration rate; ICAS, intracranial artery stenosis. 


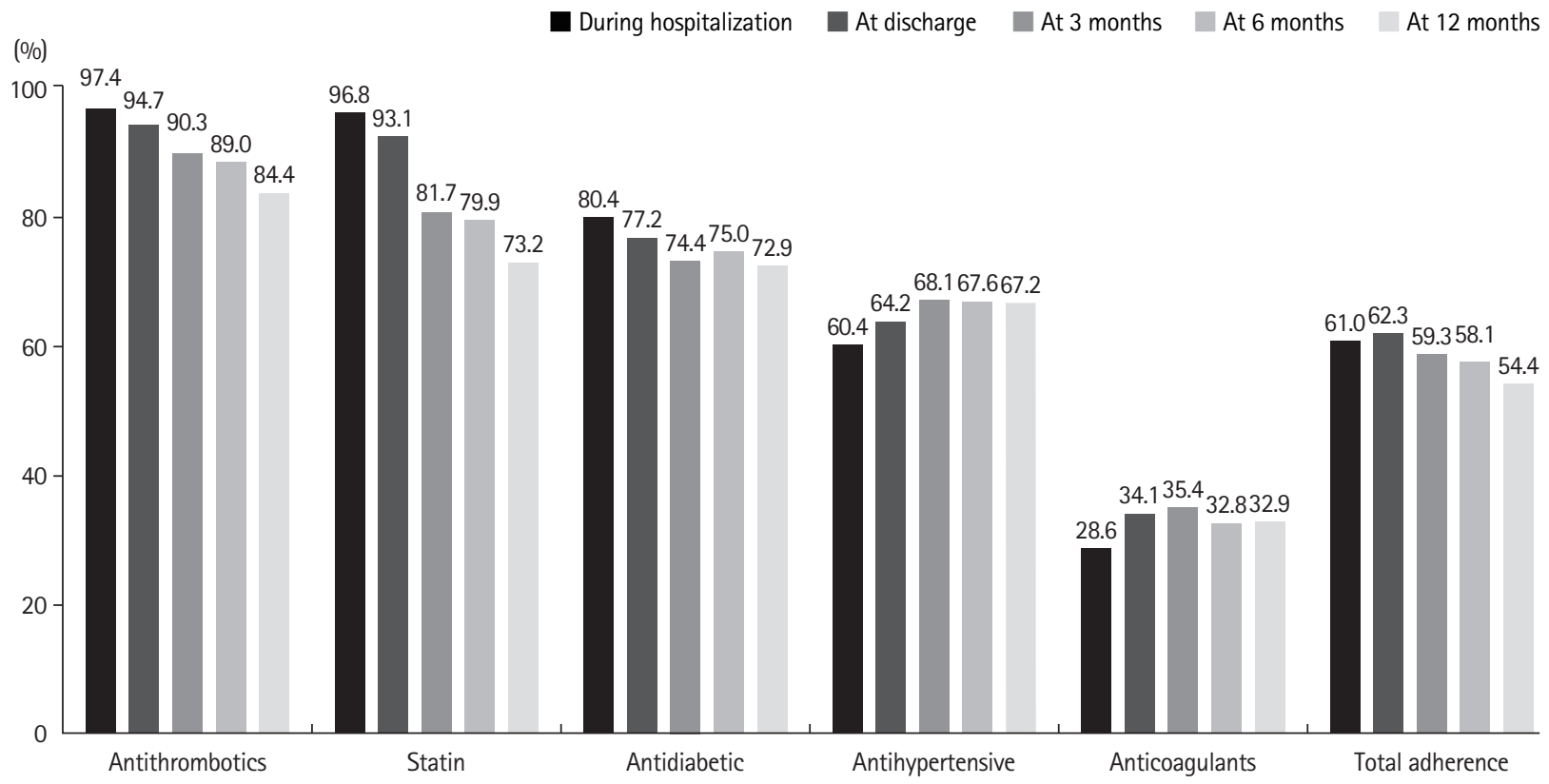

Supplementary Figure 1. Administration of secondary prevention medications for ischemic stroke and transient ischemic attack. 